\title{
The preparation of diarylacetylenes via diphenyl (benzotriazol-1-yl)(aryl)methylphosphonates
}

\author{
Alan R. Katritzky,* Boris V. Rogovoy, and Alexander Y. Mitrokhin \\ Center for Heterocyclic Compounds, Department of Chemistry, University of Florida, \\ Gainesville, FL 32611-7200, USA \\ E-mail: Katritzky@chem.ufl.edu
}

Dedicated to Professor Gerasimos (Mike) Karabatsos on his $70^{\text {th }}$ anniversary

(received 26 Feb 03; accepted 04 Jun 03; published on the web 13 Jun 03)

\begin{abstract}
A variety of differently substituted diarylacetylenes $10 \mathbf{a}-\mathbf{k}$ were obtained in good to excellent yields by a simple reaction sequence via 1-(benzotriazol-1-yl)-1-arylmethylphosphonic acid esters 8a-c and 1-(benzotriazolyl)-1,2-diarylethylenes 9a-k.
\end{abstract}

Keywords: Acetylene, diarylacetylene, benzotriazole, synthesis, phosphonate

\section{Introduction}

Acetylenes are important reagents in organic synthesis and material science. ${ }^{1}$ Many recent investigations in alkyne chemistry, particularly on diarylacetylenes, are dedicated to the synthesis of linear chain molecules with extended conjugation as potential molecular scale wires ${ }^{2}$ and light-emitting chemiluminiscent fluorophores. ${ }^{3}$ Diarylacetylenes are widely used as building blocks for the synthesis of diverse carbo- ${ }^{4}$ and heterocycles. ${ }^{5}$ Synthetic approaches to diarylacetylenes have been adapted for solid phase synthesis. ${ }^{6}$

Syntheses of diarylacetylenes have been widely investigated and summarized. ${ }^{1,7}$ Common routes for triple bond formation include (i) conversion of 1,1-diaryl-2-chloro(or bromo)ethenes in liquid ammonia in the presence of potassium amide to afford symmetrical products; ${ }^{8}$ (ii) base catalyzed elimination of $\mathrm{HX}(\mathrm{X}=\mathrm{Cl}, \mathrm{Br})$ from 2-substituted-1-aryl-1-chloroethanes formed from the corresponding benzyl ketones with an acetyl halide in the presence of $\mathrm{ZnX}_{2} / \mathrm{SiO}_{2}(\mathrm{X}=\mathrm{Cl}$, $\mathrm{Br}){ }^{9}$ and (iii) reactions of $\mathrm{N}$-benzylbenzotriazoles with arylideneamines in the presence of an excess of strong base. ${ }^{10}$ During the last decade, much attention was devoted to the synthesis of aryl and diaryl alkynes by palladium and copper catalyzed coupling of arylacetylenes. Thus, diarylacetylenes were formed (i) from terminal alkynes by the reaction with aryliodonium salts ${ }^{11}$ and with aryl or heteroaryl halides under $\operatorname{Pd}(0)$ catalysis, ${ }^{12}$ (ii) by palladium catalyzed reactions 
of silicon derivatives with aryl halides ${ }^{13}$ or triarylantimony(V)diacetals, ${ }^{14}$ and (iii) by reactions of tin derivatives with aryl iodides. ${ }^{15}$ Although many of these methods afford high yields, the availability of starting terminal arylalkynes, especially those of heteroaryl derivatives, is limited. We now report an efficient and simple protocol for the preparation of diarylacetylenes via 1(benzotriazol-1-yl)-1-arylmethylphosphonic 8 acid esters and 1-(benzotriazolyl)-1,2diarylethylenes 9.

\section{Results and Discussion}

Reactions of aldehydes with amines and phosphite esters to form $\alpha$-amino phosponates are well known. Analogous $\alpha$-amino derivatives of benzotriazole are also well known and have found a wide scope of applications. ${ }^{16}$ However, our attempt to prepare (benzotriazol-1-yl)(phenylmethyl)phosphonic acid diethyl ester $\mathbf{4}$ in a one pot reaction failed. Addition of diethyl phosphite to benzaldehyde 1 in the presence of $\mathrm{MgO}$ proceeds smoothly with the formation of 1 hydroxyphenylmethylphosphonate 2 in $90-96 \%$ yield. ${ }^{17}$ Compound 2 with methanesulfonyl chloride gave the desired methanesulfonic acid (diethoxyphosphoryl)phenylmethyl ester 3 in 70$90 \%$ yield. However, subsequent reaction of compound 3 with benzotriazole or its sodium salt gave mostly ethylbenzotriazole 5 by an Arbuzov type reaction (Scheme 1).

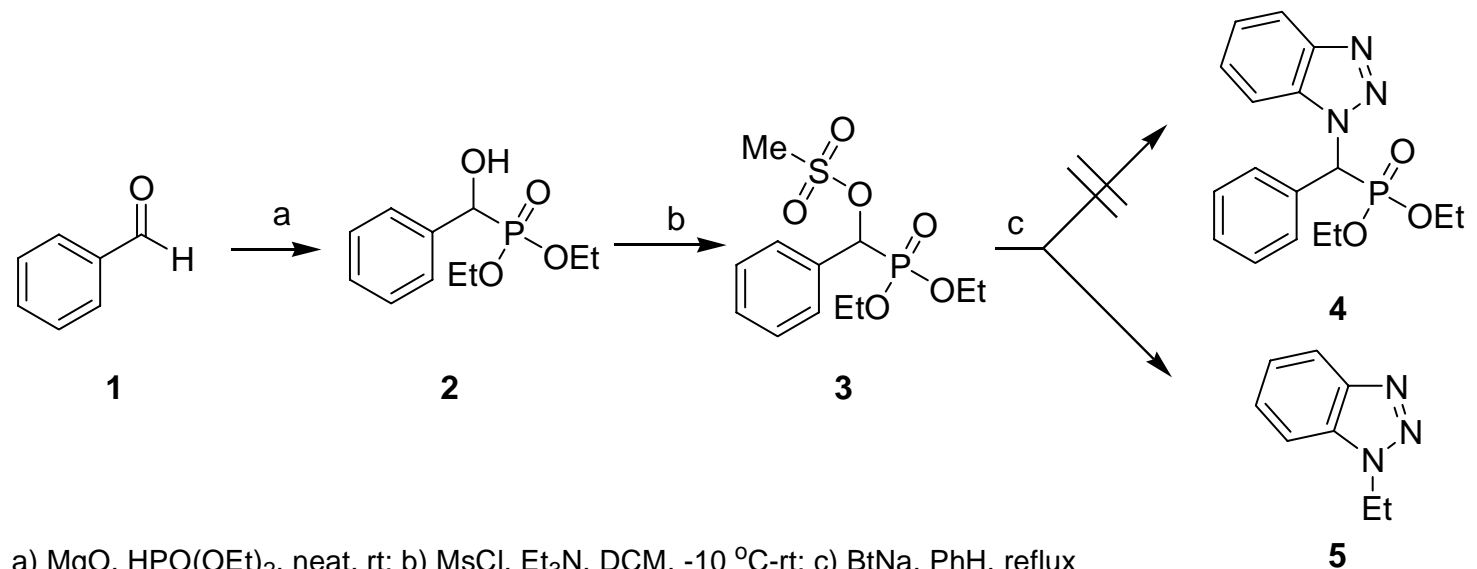

a) $\mathrm{MgO}, \mathrm{HPO}(\mathrm{OEt})_{2}$, neat, rt; b) $\mathrm{MsCl}, \mathrm{Et}_{3} \mathrm{~N}, \mathrm{DCM},-10{ }^{\circ} \mathrm{C}-\mathrm{rt}$; c) $\mathrm{BtNa}, \mathrm{PhH}$, reflux

\section{Scheme 1}

The use of diphenyl phosphite eliminates the possibility of an Arbuzov reaction and affords the analogous derivatives 8a-c in 55-75\% yield. This simple procedure succeeds with a variety substituents in the aromatic rings of products $\mathbf{8 a}-\mathbf{c}$ (Scheme 2, Table 1). 
Table 1. Isolated yields for compounds $\mathbf{6 a}-\mathbf{c}, 7 \mathbf{7}-\mathbf{c}$ and $\mathbf{8 a}-\mathbf{c}$

\begin{tabular}{cccccc}
\hline Entry & Compound & $\mathrm{Ar}^{1}$ & Yield of $\mathbf{6}$ & Yield of 7 & Yield of 8 \\
\hline 1 & $\mathbf{a}$ & $\mathrm{Ph}$ & 89 & 84 & 73 \\
2 & $\mathbf{b}$ & $4-\mathrm{CH}_{3} \mathrm{C}_{6} \mathrm{H}_{4}$ & 94 & 78 & 56 \\
3 & c & $4-\mathrm{ClC}_{6} \mathrm{H}_{4}$ & 94 & 82 & 84 \\
\hline
\end{tabular}

The obtained (benzotriazolyl)(arylmethyl)diphenylphosphonates 8a-c were lithiated by addition of $n$-butyllithium at $-78^{\circ} \mathrm{C}$, and the derived anions were condensed with aldehydes to give the benzotriazolylethylene intermediates $9 \mathbf{a}-\mathbf{k}$. As reported previously, ${ }^{10}$ derivatives $9 \mathbf{a}-\mathbf{k}$ can be easily converted into the corresponding acetylenes 10a-k by the elimination of benzotriazole in the presence of potassium tert-butoxide in $\mathrm{DMF}$ at $70-80^{\circ} \mathrm{C}$. It was found that such procedure is suitable for the elimination of a benzotriazole moiety from compounds of type 9, including heterocycle-substituted diarylethenes (Scheme 2, Table 2).

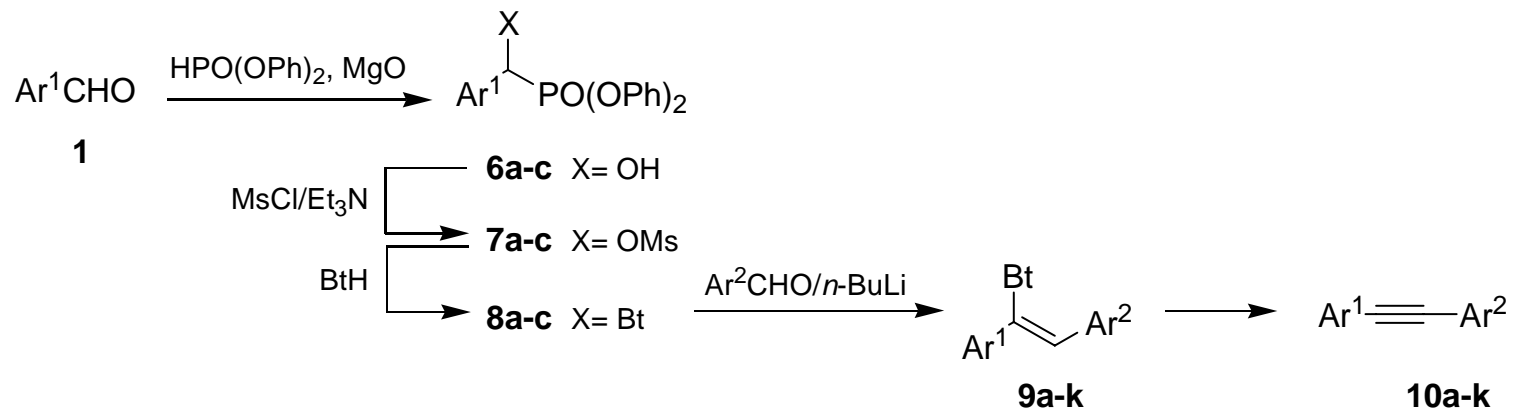

Scheme 2

Table 2. Isolated yields for compounds $9 \mathbf{a}-\mathbf{k}$ and $\mathbf{1 0 a}-\mathbf{k}$

\begin{tabular}{cccccc}
\hline Entry & Compound & Starting & $\mathrm{Ar}^{2}$ & Yield of 9 & Yield of 10 \\
\hline 1 & $\mathbf{a}$ & $\mathbf{8 a}$ & 4- $\mathrm{CH}_{3} \mathrm{C}_{6} \mathrm{H}_{4}$ & 68 & 88 \\
2 & $\mathbf{b}$ & $\mathbf{8 a}$ & $\mathrm{Ph}$ & 88 & 64 \\
3 & $\mathbf{C}$ & $\mathbf{8 a}$ & 4- $\mathrm{O}_{2} \mathrm{NC}_{6} \mathrm{H}_{4}$ & 92 & 84 \\
4 & $\mathbf{d}$ & $\mathbf{8 b}$ & 4- $\mathrm{CH}_{3} \mathrm{C}_{6} \mathrm{H}_{4}$ & 84 & 88 \\
5 & $\mathbf{e}$ & $\mathbf{8 b}$ & 1-naphthyl & 67 & 87 \\
6 & $\mathbf{f}$ & $\mathbf{8 b}$ & 2-pyridinyl & 86 & 64 \\
7 & $\mathbf{g}$ & $\mathbf{8 b}$ & 2-furyl & 78 & 62 \\
8 & $\mathbf{h}$ & $\mathbf{8 b}$ & 2-thiophenyl & 83 & 76 \\
9 & $\mathbf{i}$ & $\mathbf{8 c}$ & Ph & 63 & 48 \\
10 & $\mathbf{j}$ & $\mathbf{8 c}$ & 1-naphthyl & 73 & 75 \\
11 & $\mathbf{k}$ & $\mathbf{8 c}$ & 3-pyridinyl & 64 & 88 \\
\hline
\end{tabular}


An extension of the reported protocol was attempted for the preparation of arylalkyl derivatives. 1-(Benzotriazol-1-yl)-1-phenyl-2-cyclohexylethene $\mathbf{1 1}$ was obtained by the same reaction sequence as for diaryl derivatives. However, the elimination of benzotriazole from the compound 11 failed; no reaction was observed.

\section{Conclusions}

We have developed a convenient procedure for the preparation of diarylacetylenes via diphenyl (benzotriazolyl)(aryl)methylphosphonates 8a-c. The reaction sequence utilized standard preparative procedures with simple purification techniques and resulted in acetylenes 10a-k with a variety of substituents, including heteroaromatic moieties. All the reactions were performed under mild conditions and final diarylacetylenes 10a-k were obtained in good to excellent yields. The procedure developed may be suitable for combinatorial use.

\section{Experimental Section}

General Procedures. Melting points were determined using a capillary melting point apparatus equipped with a digital thermometer and are uncorrected. ${ }^{1} \mathrm{H}$ and ${ }^{13} \mathrm{C}$ NMR spectra were recorded on a $300 \mathrm{MHz}$ NMR spectrometer (300 and $75 \mathrm{MHz}$, respectively) using $\mathrm{CDCl}_{3}$ or DMSO- $d_{6}$ as solvents with tetramethylsilane as an internal standard. Tetrahydrofuran (THF) was distilled under nitrogen from sodium/benzophenone immediately before use. Column chromatography was conducted with silica gel grade $230-400$ mesh. All other reagents were of reagent grade and were used without purification.

\section{General procedure for the preparation of diphenyl aryl(hydroxy)methylphosphonates (6a-c)} Magnesia $(0.806 \mathrm{~g}, 0.02 \mathrm{~mol})$ was added to a mixture of diphenyl hydrogen phosphite $(4.68 \mathrm{~g}$, $0.02 \mathrm{~mol})$ and aldehyde $1 \mathrm{a}-\mathbf{c}(0.02 \mathrm{~mol})$. This mixture was stirred at room temperature for $24 \mathrm{~h}$. The resultant solid mixture was washed with DCM $(4 \times 25 \mathrm{~mL})$, and the product was isolated in a pure state by filtration through silica gel followed by recrystallization from an appropriate solvent. Due to coupling on phosphorus some carbon signals are overlapped in the ${ }^{13} \mathrm{C} N M R$ and spectra appear as described.

Diphenyl hydroxy(phenyl)methylphosphonate (6a). White needles (6.06 g, 89\%), mp 127 $128{ }^{\circ} \mathrm{C}\left(\mathrm{DCM} / \mathrm{hexanes}\right.$; lit. $\left.{ }^{18 \mathrm{a}} \mathrm{mp} 129-131{ }^{\circ} \mathrm{C}\right) ;{ }^{1} \mathrm{H}$ NMR $\left(300 \mathrm{MHz}, \mathrm{CDCl}_{3}\right): \delta 3.75(\mathrm{~s}, 1 \mathrm{H})$, $5.31(\mathrm{~d}, 1 \mathrm{H}, J=9.0 \mathrm{~Hz}), 7.00(\mathrm{~d}, 2 \mathrm{H}, J=8.4 \mathrm{~Hz}), 7.05(\mathrm{~d}, 2 \mathrm{H}, J=8.4 \mathrm{~Hz}), 7.10-7.17(\mathrm{~m}, 2 \mathrm{H})$, 7.22-7.30 (m, 4H), 7.35-7.39(m, 3H), 7.56-7.59 (m, 2H); ${ }^{13} \mathrm{C}$ NMR (75 MHz, $\left.\mathrm{CDCl}_{3}\right): \delta 69.0$ $(\mathrm{d}, J=165.9 \mathrm{~Hz}), 119.6$ (d, $J=4.0 \mathrm{~Hz}), 124.0(\mathrm{~d}, J=2.3 \mathrm{~Hz}), 126.7$ (d, $J=6.3 \mathrm{~Hz}), 127.2$ (d, $J=$ $2.9 \mathrm{~Hz}), 128.5(\mathrm{~d}, J=2.9 \mathrm{~Hz}), 135.8,149.3(\mathrm{~d}, J=9.7 \mathrm{~Hz}), 149.5(\mathrm{~d}, J=10.3 \mathrm{~Hz})$. 
Diphenyl hydroxy(4-methylphenyl)methylphosphonate (6b). White needles (6.66 g, 94\%) (DCM/hexanes), mp 100-101 ${ }^{\circ} \mathrm{C} ;{ }^{1} \mathrm{H}$ NMR $\left(300 \mathrm{MHz}, \mathrm{CDCl}_{3}\right): \delta 2.35(\mathrm{~s}, 3 \mathrm{H}), 4.35(\mathrm{br} \mathrm{s}, 1 \mathrm{H})$, $5.22(\mathrm{~d}, 1 \mathrm{H}, J=8.7 \mathrm{~Hz}), 7.01$ (d, 2H, $J=7.5 \mathrm{~Hz}), 7.07$ (d, 2H, $J=7.5 \mathrm{~Hz}), 7.10-7.18(\mathrm{~m}, 4 \mathrm{H})$, $7.20-7.30(\mathrm{~m}, 5 \mathrm{H}), 7.44(\mathrm{~d}, 1 \mathrm{H}, J=7.8 \mathrm{~Hz}) .{ }^{13} \mathrm{C}$ NMR $\left(75 \mathrm{MHz}, \mathrm{CDCl}_{3}\right) \delta 21.2,70.4(\mathrm{~d}, J=$ $161.9 \mathrm{~Hz}), 120.5(\mathrm{~d}, J=4.6 \mathrm{~Hz}), 120.6(\mathrm{~d}, J=4.0 \mathrm{~Hz}), 125.1(\mathrm{~d}, J=6.8 \mathrm{~Hz}), 127.4(\mathrm{~d}, J=6.2$ $\mathrm{Hz}), 129.1(\mathrm{~d}, J=2.8 \mathrm{~Hz}), 129.6(\mathrm{~d}, J=5.7 \mathrm{~Hz}), 132.4,138.3(\mathrm{~d}, J=3.4 \mathrm{~Hz}), 150.3(\mathrm{~d}, J=10.25$ Hz). Anal. Calcd for $\mathrm{C}_{20} \mathrm{H}_{19} \mathrm{O}_{4} \mathrm{P}$ (354.35): C, 67.79; H, 5.40. Found: $\mathrm{C}, 67.68 ; \mathrm{H}, 5.33$.

Diphenyl 4-chlorophenyl(hydroxy)methylphosphonate (6c). White needles (7.05 g, 94\%) (DCM/hexanes), mp $110-111^{\circ} \mathrm{C} ;{ }^{1} \mathrm{H}$ NMR (300 MHz, $\left.\mathrm{CDCl}_{3}\right) \delta 4.42$ (br s, $\left.1 \mathrm{H}\right), 5.24$ (d, $1 \mathrm{H}, J=$ $8.7 \mathrm{~Hz}), 6.98-7.10(\mathrm{~m}, 4 \mathrm{H}), 7.10-7.20(\mathrm{~m}, 2 \mathrm{H}), 7.20-7.38(\mathrm{~m}, 6 \mathrm{H}), 7.42-7.52(\mathrm{~m}, 2 \mathrm{H}) .{ }^{13} \mathrm{C}$ NMR $\left(75 \mathrm{MHz}, \mathrm{CDCl}_{3}\right) \delta 69.8(\mathrm{~d}, J=160.5 \mathrm{~Hz}), 120.5(\mathrm{~d}, J=4.0 \mathrm{~Hz}), 120.6(\mathrm{~d}, J=4.6 \mathrm{~Hz})$, $125.3(\mathrm{~d}, J=8.5 \mathrm{~Hz}), 128.5(\mathrm{~d}, J=2.2 \mathrm{~Hz}), 128.7$ (d, $J=6.2 \mathrm{~Hz}), 129.7$ (d, $J=6.8 \mathrm{~Hz}), 134.2$, $150.1(\mathrm{~d}, J=10.2 \mathrm{~Hz}), 150.2(\mathrm{~d}, J=9.1 \mathrm{~Hz})$. Anal. Calcd for $\mathrm{C}_{19} \mathrm{H}_{16} \mathrm{ClO}_{4} \mathrm{P}$ (374.76): C, 60.89; H, 4.30; Found: C, 60.66; H, 4.65 .

General procedure for the preparation of aryl(diphenoxyphosphoryl)methyl methanesulfonates $(7 a-c)$

To a solution of compound $6(0.05 \mathrm{~mol})$ and triethylamine $(8.10 \mathrm{~g}, 0.08 \mathrm{~mol})$ in DCM $(100 \mathrm{~mL})$ at $-10{ }^{\circ} \mathrm{C}$ was added dropwise methanesulfonyl chloride $(5.73 \mathrm{~g}, 0.05 \mathrm{~mol})$ under vigorous stirring. Stirring for an additional 10-15 min completed the reaction. Then the reaction mixture was washed with ice water, cold $10 \%$ hydrochloric acid, saturated sodium bicarbonate solution and brine. After drying over $\mathrm{MgSO}_{4}$, evaporation of solvent gave crude product, which was recrystallized from an appropriate solvent. Due to coupling on phosphorus some carbon signals are overlapped in the ${ }^{13} \mathrm{C}$ NMR and spectra appear as described.

(Phenyl)(diphenoxyphosphoryl)methyl methanesulfonate (7a). White needles (17.5 g, 84\%) (DCM/ hexanes), mp 75-76 ${ }^{\circ} \mathrm{C} ;{ }^{1} \mathrm{H}$ NMR $\left(300 \mathrm{MHz}, \mathrm{CDCl}_{3}\right): \delta 2.87$ (s, 3H), 6.11 (d, $1 \mathrm{H}, J=15$ $\mathrm{Hz}), 7.0(\mathrm{~d}, 2 \mathrm{H}, J=7.2 \mathrm{~Hz}), 7.07-7.22(\mathrm{~m}, 4 \mathrm{H}), 7.22-7.38(\mathrm{~m}, 4 \mathrm{H}), 7.41-7.48(\mathrm{~m}, 3 \mathrm{H}), 7.58-$ $7.68(\mathrm{~m}, 2 \mathrm{H}) .{ }^{13} \mathrm{C} \mathrm{NMR}\left(75 \mathrm{MHz}, \mathrm{CDCl}_{3}\right): \delta 39.6,76.9(\mathrm{~d}, J=175.3 \mathrm{~Hz}), 120.3(\mathrm{~d}, J=4.6 \mathrm{~Hz})$, $120.4(\mathrm{~d}, J=4.6 \mathrm{~Hz}), 125.5,128.5$ (d, $J=6.2 \mathrm{~Hz}), 129.0$ (d, $J=1.7 \mathrm{~Hz}), 129.7$ (d, $J=1.7 \mathrm{~Hz})$, $130.1(\mathrm{~d}, J=2.8 \mathrm{~Hz}), 131.0,149.7(\mathrm{~d}, J=9.1 \mathrm{~Hz}), 149.8(\mathrm{~d}, J=9.7 \mathrm{~Hz})$. Anal. Calcd for $\mathrm{C}_{20} \mathrm{H}_{19} \mathrm{O}_{6} \mathrm{PS}$ (418.41): C, 57.41; H, 4.58; Found: C, 57.42; H, 4.38.

(Diphenoxyphosphoryl)(4-methylphenyl)methyl methanesulfonate (7b). White needles (16.86 g, 78\%) (DCM/hexanes), mp 133-134 ${ }^{\circ} \mathrm{C} ;{ }^{1} \mathrm{H}$ NMR $\left(300 \mathrm{MHz}, \mathrm{CDCl}_{3}\right): \delta 2.38(\mathrm{~s}, 3 \mathrm{H})$, $2.84(\mathrm{~s}, 3 \mathrm{H}), 6.06(\mathrm{~d}, 1 \mathrm{H}, J=14.7 \mathrm{~Hz}), 7.01(\mathrm{~d}, 2 \mathrm{H}, J=7.5 \mathrm{~Hz}), 7.14-7.31(\mathrm{~m}, 10 \mathrm{H}), 7.52(\mathrm{dd}$, $\left.2 \mathrm{H}, J_{1}=7.2 \mathrm{~Hz}, J_{2}=1.5 \mathrm{~Hz}\right) .{ }^{13} \mathrm{C}$ NMR $\left(75 \mathrm{MHz}, \mathrm{CDCl}_{3}\right): \delta 21.3,39.8,77.0(\mathrm{~d}, J=176.5 \mathrm{~Hz})$, $120.4(\mathrm{~d}, J=4.5 \mathrm{~Hz}), 120.5$ (d, $J=3.4 \mathrm{~Hz}), 125.6,127.8,128.6$ (d, $J=6.8 \mathrm{~Hz}), 128.7$ (d, $J=3.5$ Hz), 129.7 (d, $J=2.3 \mathrm{~Hz}), 129.8$ (d, $J=3.5 \mathrm{~Hz}), 140.3,149.8$ (d, $J=9.7 \mathrm{~Hz}), 150.0$ (d, $J=10.3$ $\mathrm{Hz}$ ). Compound was used without purification for the further transformations.

Diphenoxyphosphoryl(4-chlorophenyl)methyl methanesulfonate (7c). White needles (18.56 g, 82\%) (DCM/hexanes), mp 100-102 ${ }^{\circ} \mathrm{C} ;{ }^{1} \mathrm{H}$ NMR (300 MHz, $\left.\mathrm{CDCl}_{3}\right): \delta 2.94(\mathrm{~s}, 3 \mathrm{H})$, 
$6.08(\mathrm{~d}, 1 \mathrm{H}, J=15 \mathrm{~Hz}), 7.02(\mathrm{~d}, 2 \mathrm{H}, J=7.8 \mathrm{~Hz}), 7.11(\mathrm{~d}, 2 \mathrm{H}, J=7.8 \mathrm{~Hz}), 7.20(\mathrm{t}, 2 \mathrm{H}, J=6.9$ $\mathrm{Hz}), 7.22-7.36(\mathrm{~m}, 5 \mathrm{H}), 7.42(\mathrm{~d}, 2 \mathrm{H}, J=8.4 \mathrm{~Hz}), 7.58(\mathrm{~d}, 2 \mathrm{H}, J=6.9 \mathrm{~Hz}) .{ }^{13} \mathrm{C}$ NMR $(75 \mathrm{MHz}$, $\left.\mathrm{CDCl}_{3}\right): \delta 39.7,76.0(\mathrm{~d}, J=174.2 \mathrm{~Hz}), 120.2(\mathrm{~d}, J=4.0 \mathrm{~Hz}), 120.3(\mathrm{~d}, J=4.0 \mathrm{~Hz}), 125.7,129.3$ (d, $J=2.3 \mathrm{~Hz}), 129.7,129.9,136.2(\mathrm{~d}, J=3.4 \mathrm{~Hz}), 149.6$ (d, $J=9.2 \mathrm{~Hz}), 149.8$ (d, $J=9.7 \mathrm{~Hz})$. Anal. Calcd for $\mathrm{C}_{20} \mathrm{H}_{18} \mathrm{ClO}_{6} \mathrm{PS}$ (452.85): C, 53.05; H, 4.01; Found: C, 53.26; H, 4.07.

General procedure for the preparation of diphenyl (1H-benzotriazol-1-yl)(aryl)methylphosphonate 8a-c

A mixture of (diphenoxyphosphoryl)(aryl)methyl methanesulfonate 7a-c $(0.025 \mathrm{~mol})$ with benzotriazole $(5.96 \mathrm{~g}, 0.05 \mathrm{~mol})$ in toluene $(150 \mathrm{~mL})$ was refluxed for $48 \mathrm{~h}$. The mixture was cooled down to room temperature, washed with sat. $\mathrm{Na}_{2} \mathrm{CO}_{3}$, water, brine and dried over $\mathrm{Na}_{2} \mathrm{SO}_{4}$. The solvent was removed in vacuo to give a brown residue, which was purified on silica (eluent hexanes:EtOAc 3:1) to give the desired products 8a-c.

Diphenyl (1H-benzotriazol-1-yl)(phenyl)methylphosphonate (8a). White needles $(8.06 \mathrm{~g}$, $73 \%$ ) (ethyl acetate/hexanes), mp 133-134 ${ }^{\circ} \mathrm{C} ;{ }^{1} \mathrm{H}$ NMR (300 MHz, $\mathrm{CDCl}_{3}$ ): $\delta 6.74$ (d, $1 \mathrm{H}, J=$ $23.7 \mathrm{~Hz}), 6.85(\mathrm{~d}, 2 \mathrm{H}, J=8.1 \mathrm{~Hz}), 6.98(\mathrm{~d}, 2 \mathrm{H}, J=8.1 \mathrm{~Hz}), 6.99-7.30(\mathrm{~m}, 6 \mathrm{H}), 7.32-7.45(\mathrm{~m}$, $5 \mathrm{H}), 7.67(\mathrm{~d}, 1 \mathrm{H}, J=8.4 \mathrm{~Hz}), 7.68-7.73(\mathrm{~m}, 2 \mathrm{H}), 8.07(\mathrm{~d}, 1 \mathrm{H}, J=8.1 \mathrm{~Hz}) .{ }^{13} \mathrm{C}$ NMR $(75 \mathrm{MHz}$, $\left.\mathrm{CDCl}_{3}\right): \delta 60.0(\mathrm{~d}, J=158.2 \mathrm{~Hz}), 110.3,120.1(\mathrm{~d}, J=4.6 \mathrm{~Hz}), 120.3(\mathrm{~d}, J=4.0 \mathrm{~Hz}), 124.3$, $125.5(\mathrm{~d}, J=11.3 \mathrm{~Hz}), 128.0,129.2,129.6(\mathrm{~d}, J=11.9 \mathrm{~Hz}), 130.2(\mathrm{~d}, J=6.8 \mathrm{~Hz}), 132.6(\mathrm{~d}, J=$ $3.4 \mathrm{~Hz}), 135.5(\mathrm{~d}, J=2.8 \mathrm{~Hz}), 146.0,149.6(\mathrm{~d}, J=10.8 \mathrm{~Hz})$. Anal. Calcd for $\mathrm{C}_{25} \mathrm{H}_{20} \mathrm{~N}_{3} \mathrm{O}_{3} \mathrm{P}$ (441.43): C, 68.02; H, 4.57; N, 9.52. Found: C, 67.93; H, 4.63; N, 9.52.

Diphenyl (1H-benzotriazol-1-yl)(4-methylphenyl)methylphosphonate (8b). White needles (6.38 g, 56\%) (ethyl acetate/hexanes), mp 148-149 ${ }^{\circ} \mathrm{C} ;{ }^{1} \mathrm{H}$ NMR (300 MHz, $\mathrm{CDCl}_{3}$ ): $\delta 2.33$ (s, $3 \mathrm{H}), 6.71(\mathrm{~d}, 1 \mathrm{H}, J=23.4 \mathrm{~Hz}), 6.85(\mathrm{~d}, 2 \mathrm{H}, J=8.1 \mathrm{~Hz}), 7.00(\mathrm{~d}, 2 \mathrm{H}, J=8.1 \mathrm{~Hz}), 7.03-7.29(\mathrm{~m}$, $8 \mathrm{H}), 7.32-7.48(\mathrm{~m}, 2 \mathrm{H}), 7.60(\mathrm{~d}, 1 \mathrm{H}, J=7.5 \mathrm{~Hz}), 7.66(\mathrm{~d}, 1 \mathrm{H}, J=8.1 \mathrm{~Hz}), 8.05(\mathrm{~d}, 1 \mathrm{H}, J=8.4$ Hz). ${ }^{13} \mathrm{C}$ NMR (75 MHz, $\left.\mathrm{CDCl}_{3}\right): \delta 21.0,60.4(\mathrm{~d}, J=158.3 \mathrm{~Hz}), 110.6,119.9,120.1(\mathrm{~d}, J=4.5$ $\mathrm{Hz}), 120.4(\mathrm{~d}, J=4.0 \mathrm{~Hz}), 124.1,125.4(\mathrm{~d}, J=11.4 \mathrm{~Hz}), 127.7,128.1,128.8(\mathrm{~d}, J=7.4 \mathrm{~Hz})$, $129.5,129.6(\mathrm{~d}, J=2.8 \mathrm{~Hz}), 132.6(\mathrm{~d}, J=3.4 \mathrm{~Hz}), 139.4(\mathrm{~d}, J=2.3 \mathrm{~Hz}), 146.0,149.7(\mathrm{~d}, J=2.8$ $\mathrm{Hz}), 149.8\left(\mathrm{~d}, J=2.8 \mathrm{~Hz}\right.$ ). Anal. Calcd for $\mathrm{C}_{26} \mathrm{H}_{22} \mathrm{~N}_{3} \mathrm{O}_{3} \mathrm{P}$ (455.46): C, 68.57; H, 4.87; N, 9.23. Found: C, 68.59; H, 5.12; N, 9.10.

Diphenyl (1H-benzotriazol-1-yl)(4-chlorophenyl)methylphosphonate (8c). White prisms (9.99 g, 84\%) (ethyl acetate/hexanes), mp 113-114 ${ }^{\circ} \mathrm{C} ;{ }^{1} \mathrm{H} \mathrm{NMR}\left(300 \mathrm{MHz}, \mathrm{CDCl}_{3}\right): \delta 6.69$ (d, $1 \mathrm{H}, J=23.7 \mathrm{~Hz}), 6.86(\mathrm{~d}, 2 \mathrm{H}, J=8.1 \mathrm{~Hz}), 6.99(\mathrm{~d}, 2 \mathrm{H}, J=8.0 \mathrm{~Hz}), 7.04-7.12(\mathrm{~m}, 1 \mathrm{H}), 7.12$ $7.20(\mathrm{~m}, 3 \mathrm{H}), 7.24-7.30(\mathrm{~m}, 2 \mathrm{H}), 7.32-7.40(\mathrm{~m}, 3 \mathrm{H}), 7.40-7.49(\mathrm{~m}, 1 \mathrm{H}), 7.64(\mathrm{~m}, 3 \mathrm{H}), 8.08(\mathrm{~d}$, $1 \mathrm{H}, J=8.4 \mathrm{~Hz}) .{ }^{13} \mathrm{C}$ NMR $\left(75 \mathrm{MHz}, \mathrm{CDCl}_{3}\right): \delta 59.9(\mathrm{~d}, J=158.2 \mathrm{~Hz}), 110.3,120.1(\mathrm{~d}, J=4.0$ $\mathrm{Hz}), 120.2,120.4(\mathrm{~d}, J=4.0 \mathrm{~Hz}), 124.4,125.6$ (d, $J=12.4 \mathrm{~Hz}), 128.1,129.3,129.7$ (d, $J=11.9$ $\mathrm{Hz}), 130.2(\mathrm{~d}, J=6.8 \mathrm{~Hz}), 132.6(\mathrm{~d}, J=3.4 \mathrm{~Hz}), 135.6(\mathrm{~d}, J=2.3 \mathrm{~Hz}) 140.6,149.7(\mathrm{~d}, J=9.0$ Hz). Anal. Calcd for $\mathrm{C}_{25} \mathrm{H}_{19} \mathrm{ClN}_{3} \mathrm{O}_{3} \mathrm{P}$ (475.88): C, 63.10; H, 4.02; N, 8.83. Found: C, 62.99; H, $3.92 ; \mathrm{N}, 8.71$. 


\section{General procedure for the preparation of 1,2-diaryl-1-(benzotriazol-1-yl)ethenes 9a-k and compound 11}

To a solution of diphenyl $1 H$-benzotriazol-1-yl(aryl)methylphosphonate 8a-c $(5 \mathrm{mmol})$ in $4 \mathrm{~mL}$ of dry THF at $-78^{\circ} \mathrm{C}$ under $\mathrm{N}_{2}, n$-BuLi $(5.1 \mathrm{mmol})$ was added. The mixture was stirred at $-78^{\circ} \mathrm{C}$ for $10 \mathrm{~min}$, then the respective aldehyde $(5 \mathrm{mmol})$ was added dropwise and the reaction mixture was stirred at $-78^{\circ} \mathrm{C}$ for $10 \mathrm{~min}$. The acetone-dry ice bath was removed and the mixture was allowed to warm up to room temperature over $40 \mathrm{~min}$ and quenched with sat. $\mathrm{NH}_{4} \mathrm{Cl}$. The mixture was twice extracted with EtOAc, the combined extracts were washed with sat. $\mathrm{NH}_{4} \mathrm{Cl}$, brine and dried over $\mathrm{Na}_{2} \mathrm{SO}_{4}$. The solvent was removed under reduced pressure to give a brown residue, which was purified on silica (hexanes/EtOAc $=3: 1$ as eluent) to give the desired products 9a-k.

1-[2-(4-Methylphenyl)-1-phenylethenyl]-1H-benzotriazole (9a). White needles (1.06 g, 68\%) (hexanes), mp 118-119 ${ }^{\circ} \mathrm{C} ;{ }^{1} \mathrm{H}$ NMR $\left(300 \mathrm{MHz}, \mathrm{CDCl}_{3}\right): \delta 2.32$ (s, 3H), $6.80(\mathrm{~d}, 1 \mathrm{H}, J=8.4$ $\mathrm{Hz}), 7.03(\mathrm{~d}, 2 \mathrm{H}, J=7.8 \mathrm{~Hz}), 7.12(\mathrm{~d}, 2 \mathrm{H}, J=7.8 \mathrm{~Hz}), 7.20-7.41(\mathrm{~m}, 8 \mathrm{H}), 8.08$ (d, $1 \mathrm{H}, J=8.1$ $\mathrm{Hz}) .{ }^{13} \mathrm{C}$ NMR $\left(75 \mathrm{MHz}, \mathrm{CDCl}_{3}\right): \delta 21.3,30.9,111.4,120.0,124.0,126.0,127.5,129.1,129.5$, 129.7, 131.4, 132.7, 133.8, 134.4, 138.0, 146.3. Anal. Calcd for $\mathrm{C}_{21} \mathrm{H}_{17} \mathrm{~N}_{3}$ (311.39): C, 81.00; $\mathrm{H}$, 5.50; N, 13.49. Found: C, 80.62; H, 5.37; N, 13.42 .

1-(Benzotriazol-1-yl)-1,2-diphenylethylene (9b). White prisms (1.31 g, 88\%) (hexanes), mp 111-112 ${ }^{\circ} \mathrm{C}$ (lit mp $\left.{ }^{10 \mathrm{a}} 111-113{ }^{\circ} \mathrm{C}\right) ;{ }^{1} \mathrm{H}$ NMR (300 MHz, $\left.\mathrm{CDCl}_{3}\right): \delta 6.79(\mathrm{~d}, 1 \mathrm{H}, J=7.8 \mathrm{~Hz})$, $7.18-7.48(\mathrm{~m}, 13 \mathrm{H}), 8.08(\mathrm{~d}, 1 \mathrm{H}, J=8.1 \mathrm{~Hz}) .{ }^{13} \mathrm{C} \mathrm{NMR}\left(75 \mathrm{MHz}, \mathrm{CDCl}_{3}\right): \delta 111.4,120.1$, 124.1, 125.9, 127.6, 128.0, 128.3, 129.1, 129.6, 129.7, 129.8, 132.7, 133.6, 134.3, 135.2, 146.3. Anal. Calcd for $\mathrm{C}_{20} \mathrm{H}_{15} \mathrm{~N}_{3}$ (297.36): C, 80.78; H, 5.08; N, 14.13. Found: C, 80.74; H, 5.24; N, 14.28 .

1-[2-(4-Nitrophenyl)-1-phenylethenyl]-1H-benzotriazole (9c). Yellow needles (1.57 g, 92\%) (hexanes) mp 167-168 ${ }^{\circ} \mathrm{C} ;{ }^{1} \mathrm{H} \mathrm{NMR}\left(300 \mathrm{MHz}, \mathrm{CDCl}_{3}\right): \delta 6.60(\mathrm{~d}, 1 \mathrm{H}, J=8.4 \mathrm{~Hz}), 7.22-7.56$ $(\mathrm{m}, 10 \mathrm{H}), 8.07-8.13(\mathrm{~m}, 3 \mathrm{H}),{ }^{13} \mathrm{C} \mathrm{NMR}\left(75 \mathrm{MHz}, \mathrm{CDCl}_{3}\right): \delta 111.5,120.4,122.2,123.7,124.4$, $128.0,129.6,130.0,130.2,130.6,132.4,132.5,138.6,141.4,146.5,146.7$. Anal. Calcd for $\mathrm{C}_{20} \mathrm{H}_{14} \mathrm{~N}_{4} \mathrm{O}_{2}$ (342.36): C, 70.17; H, 4.12; N, 16.36. Found: C, 70.32; H, 4.28; N, 16.11 .

1-[1,2-Bis(4-methylphenyl)ethenyl]-1H-benzotriazole (9d). Colorless oil $(1.37 \mathrm{~g}, 84 \%) .{ }^{1} \mathrm{H}$ NMR $\left(300 \mathrm{MHz}, \mathrm{CDCl}_{3}\right): \delta 2.32(\mathrm{~s}, 3 \mathrm{H}), 2.39(\mathrm{~s}, 3 \mathrm{H}), 6.83(\mathrm{~d}, 1 \mathrm{H}, J=7.8 \mathrm{~Hz}), 7.03(\mathrm{~d}, 2 \mathrm{H}, J=$ $8.1 \mathrm{~Hz}), 7.10-7.38(\mathrm{~m}, 9 \mathrm{H}), 8.08(\mathrm{~d}, 1 \mathrm{H}, J=8,1 \mathrm{~Hz}) .{ }^{13} \mathrm{C} \mathrm{NMR}\left(75 \mathrm{MHz}, \mathrm{CDCl}_{3}\right): \delta 21.3,21.5$, $111.5,120.0$, 124.0, 125.5, 127.4, 129.0, 129.4, 129.6, 129.8, 130.8, 131.6, 132.7, 134.5, 137.9, 139.6, 146.3. This compound was used as a crude material without isolation.

1-[1-(4-Methylphenyl)-2-(1-naphthyl)ethenyl]-1H-benzotriazole (9e). White needles (1.21 g, 67\%) (hexanes), mp 122-123 ${ }^{\circ} \mathrm{C} ;{ }^{1} \mathrm{H}$ NMR (300 MHz, $\left.\mathrm{CDCl}_{3}\right): \delta 2.31(\mathrm{~s}, 3 \mathrm{H}), 6.88(\mathrm{~d}, 1 \mathrm{H}, J=$ 8.1 Hz), 6.97-7.04 (m, 4H), 7.21-7.39 (m, 4H), 7.50-7.55 (m, 2H), $7.71(\mathrm{~s}, 1 \mathrm{H}), 7.78(\mathrm{~d}, 1 \mathrm{H}, J=$ $8.1 \mathrm{~Hz}), 7.86-7.91(\mathrm{~m}, 1 \mathrm{H}), 8.10(\mathrm{~d}, 1 \mathrm{H}, J=4.2 \mathrm{~Hz}), 8.14(\mathrm{~d}, 1 \mathrm{H}, J=8.1 \mathrm{~Hz}),{ }^{13} \mathrm{C}$ NMR $(300$ $\left.\mathrm{MHz}, \mathrm{CDCl}_{3}\right): \delta 21.4,111.6,120.1,123.6,124.1,124.3,125.4,126.1,126.5,127.5,127.9$, $128.3,128.6,129.4,130.4,132.0,132.2,132.9,133.5,137.0,139.4,146.4$. Anal. Calcd for $\mathrm{C}_{25} \mathrm{H}_{19} \mathrm{~N}_{3}$ (361.45): C, 83.08; H, 5.30; N, 11.63. Found: C, 82.78; H, 5.42; N, 11.53. 
1-[1-(4-Methylphenyl)-2-(2-pyridinyl)ethenyl]-1H-benzotriazole (9f). White needles (1.34 g, 84\%) (hexanes), mp 105-106 ${ }^{\circ} \mathrm{C} ;{ }^{1} \mathrm{H}$ NMR $\left(300 \mathrm{MHz}, \mathrm{CDCl}_{3}\right): \delta 2.40(\mathrm{~s}, 3 \mathrm{H}), 6.81(\mathrm{~d}, 1 \mathrm{H}, J=$ $7.8 \mathrm{~Hz}), 7.05-7.23(\mathrm{~m}, 6 \mathrm{H}), 7.25-7.38(\mathrm{~m}, 3 \mathrm{H}), 7.45(\mathrm{t}, 1 \mathrm{H}, J=8.1 \mathrm{~Hz}), 8.09$ (d, $1 \mathrm{H}, J=8.1$ $\mathrm{Hz}), 8.61(\mathrm{~d}, 1 \mathrm{H}, J=4.5 \mathrm{~Hz}) .{ }^{13} \mathrm{C} \mathrm{NMR}\left(75 \mathrm{MHz}, \mathrm{CDCl}_{3}\right): \delta 21.5,111.6,120.2,122.1,124.2$, $124.3,127.7,129.7,129.8,130.1,132.7,135.7,138.5,140.3,146.5,149.8,154.5$. Anal. Calcd for $\mathrm{C}_{20} \mathrm{H}_{16} \mathrm{~N}_{4}(312.38)$ : C, 76.90; H, 5.16; N, 17.94. Found: C, 76.76; H, 5.25; N, 17.91.

1-[2-(2-Furyl)-1-(4-methylphenyl)ethenyl]-1H-benzotriazole (9g). White needles (1.17 g, 78\%), mp 127-128 ${ }^{\circ} \mathrm{C} ;{ }^{1} \mathrm{H}$ NMR (300 MHz, $\left.\mathrm{CDCl}_{3}\right): \delta 2.44(\mathrm{~s}, 3 \mathrm{H}), 6.22(\mathrm{~d}, 1 \mathrm{H}, J=3 \mathrm{~Hz}), 6.34$ $(\mathrm{s}, 1 \mathrm{H}), 6.74(\mathrm{~d}, 1 \mathrm{H}, J=8.1 \mathrm{~Hz}), 7.12(\mathrm{~s}, 1 \mathrm{H}), 7.20-7.39(\mathrm{~m}, 7 \mathrm{H}), 8.06(\mathrm{~d}, 1 \mathrm{H}, J=7.8 \mathrm{~Hz}) .{ }^{13} \mathrm{C}$ NMR $\left(75 \mathrm{MHz}, \mathrm{CDCl}_{3}\right): \delta 21.5,111.5,111.6,113.2,120.0,124.0,127.5,128.3,129.5,129.6$, 130.7, 132.6, 133.0, 140.0, 142.7, 146.4, 150.1. Anal. Calcd for $\mathrm{C}_{19} \mathrm{H}_{15} \mathrm{~N}_{3} \mathrm{O}$ (301.35): C, 75.73; H, 5.02; N, 13.94. Found: C, 75.96; H, 5.20; N, 13.50 .

1-[1-(4-Methylphenyl)-2-(2-thienyl)ethenyl]-1H-benzotriazole (9h). White needles (1.32 g, 83\%), mp 140-141 ${ }^{\circ} \mathrm{C} ;{ }^{1} \mathrm{H}$ NMR (300 MHz, $\left.\mathrm{CDCl}_{3}\right): \delta 2.46(\mathrm{~s}, 3 \mathrm{H}), 6.74(\mathrm{~d}, 1 \mathrm{H}, J=7.8 \mathrm{~Hz})$, $6.94\left(\mathrm{dd}, 1 \mathrm{H}, J_{1}=3.9 \mathrm{~Hz}, J_{2}=1.2 \mathrm{~Hz}\right), 7.01(\mathrm{~d}, 1 \mathrm{H}, J=3.3 \mathrm{~Hz}), 7.14(\mathrm{~d}, 1 \mathrm{H}, J=5.1 \mathrm{~Hz})$, $7.21-7.27(\mathrm{~m}, 4 \mathrm{H}), 7.40(\mathrm{~d}, 2 \mathrm{H}, J=8.1 \mathrm{~Hz}), 7.51(\mathrm{~s}, 1 \mathrm{H}), 8.06(\mathrm{~d}, 1 \mathrm{H}, J=7.8 \mathrm{~Hz}) .{ }^{13} \mathrm{C} \mathrm{NMR}(75$ $\left.\mathrm{MHz}, \mathrm{CDCl}_{3}\right): \delta 21.6,111.5,118.1,120.1,124.0,126.6,126.7,127.5,130.0,130.1,130.2$, 132.5, 133.4, 137.6, 140.5, 146.4. Anal. Calcd for $\mathrm{C}_{19} \mathrm{H}_{15} \mathrm{~N}_{3} \mathrm{~S}$ (317.42): C, 71.90; H, 4.76; N, 13.24. Found: C, 72.19; H, 4.97; N, 13.29.

1-(Benzotriazol-1-yl)-1-(4-chlorophenyl)-2-phenylethylene (9i). Yellow oil (1.05 g, 63\%). ${ }^{1} \mathrm{H}$ NMR $\left(300 \mathrm{MHz}, \mathrm{CDCl}_{3}\right): \delta 6.88\left(\mathrm{dd}, 1 \mathrm{H}, J_{1}=5.7 \mathrm{~Hz}, J_{2}=2.7 \mathrm{~Hz}\right) ; 7.20-7.28(\mathrm{~m}, 7 \mathrm{H}) ; 7.28-$ $7.42(\mathrm{~m}, 6 \mathrm{H}) ; 8.11\left(\mathrm{dd}, 1 \mathrm{H}, J_{1}=6.0 \mathrm{~Hz}, J_{2}=1.8 \mathrm{~Hz}\right) ;{ }^{13} \mathrm{C} \mathrm{NMR}\left(75 \mathrm{MHz}, \mathrm{CDCl}_{3}\right): \delta 111.2$, 120.3, 124.2, 126.6, 127.8, 128.3, 129.4, 129.4, 129.5, 129.9, 131.1, 132.1, 132.6, 134.1, 135.6. This compound was used as a crude material without isolation.

1-[1-(4-Chlorophenyl)-2-(1-naphthyl)ethenyl]-1H-benzotriazole (9j). White prisms (1.39 g, 73\%), mp 137-138 ${ }^{\circ} \mathrm{C}$; ${ }^{1} \mathrm{H}$ NMR (300 MHz, $\left.\mathrm{CDCl}_{3}\right): \delta 6.95(\mathrm{~d}, 1 \mathrm{H}, J=7.8 \mathrm{~Hz}), 7.06(\mathrm{~d}, 2 \mathrm{H}, J=$ $8.1 \mathrm{~Hz}), 7.17(\mathrm{~d}, 2 \mathrm{H}, J=8.4 \mathrm{~Hz}), 7.22-7.44(\mathrm{~m}, 4 \mathrm{H}), 7.48-7.58(\mathrm{~m}, 2 \mathrm{H}), 7.74-7.85(\mathrm{~m}, 2 \mathrm{H})$, 7.85-7.94 (m, 1H), 8.02-8.12 (m, 1H), $8.15(\mathrm{~d}, 1 \mathrm{H}, J=7.5 \mathrm{~Hz}) .{ }^{13} \mathrm{C}$ NMR $\left(75 \mathrm{MHz}, \mathrm{CDCl}_{3}\right): \delta$ $111.2,120.3,124.2,124.4,125.0,125.4,126.3,126.7,127.9,128.0,128.7,129.1,130.9,131.5$, 132.0, 132.0, 132.8, 133.6, 135.3, 135.9, 146.4. Anal. Calcd for $\mathrm{C}_{24} \mathrm{H}_{16} \mathrm{ClN}_{3}$ (381.87): C, 75.49; H, 4.22; N, 11.00. Found: C, 75.84; H, 4.31; N, 10.64 .

1-[1-(4-Chlorophenyl)-2-(3-pyridinyl)ethenyl]-1H-benzotriazole (9k). Oil (1.06 g, 64\%). ${ }^{1} \mathrm{H}$ NMR (300 MHz, $\left.\mathrm{CDCl}_{3}\right): \delta 6.78(\mathrm{~d}, 1 \mathrm{H}, J=8.1 \mathrm{~Hz}), 7.14-7.22(\mathrm{~m}, 1 \mathrm{H}), 7.22-7.90(\mathrm{~m}, 3 \mathrm{H})$, $7.90-7.43(\mathrm{~m}, 4 \mathrm{H}), 7.48(\mathrm{~d}, 1 \mathrm{H}, J=8.1 \mathrm{~Hz}), 8.12(\mathrm{~d}, 1 \mathrm{H}, J=7.5 \mathrm{~Hz}), 8.43-8.54(\mathrm{~m}, 2 \mathrm{H}) .{ }^{13} \mathrm{C}$ NMR $\left(75 \mathrm{MHz}, \mathrm{CDCl}_{3}\right): \delta 111.1,120.3,121.9,123.3,124.4,128.0,129.8,130.3,131.0,131.1$, $132.3,136.0,136.1,136.3,146.4,148.8,150.5$. This compound was used as a crude material without isolation.

1-[(Z)-2-Cyclohexyl-1-phenylethenyl]-1H-1,2,3-benzotriazole (11). White needles (0.97 g, 64\%), mp 111-112 ${ }^{\circ} \mathrm{C} ;{ }^{1} \mathrm{H}$ NMR $\delta 1.10-1.44(\mathrm{~m}, 5 \mathrm{H}), 1.60-1.88(\mathrm{~m}, 5 \mathrm{H}), 2.44-2.62(\mathrm{~m}, 1 \mathrm{H})$, $6.18(\mathrm{~d}, 1 \mathrm{H}, J=10.8 \mathrm{~Hz}), 6.89(\mathrm{~m}, 1 \mathrm{H}), 7.20-7.32(\mathrm{~m}, 4 \mathrm{H}), 7.33-7.49(\mathrm{~m}, 3 \mathrm{H}), 8.05$ (m, 1H). 
${ }^{13} \mathrm{C}$ NMR $\delta 25.4,25.7,33.1,37.0,111.0,119.9,123.8,127.3,128.7,128.8,129.0,132.8,133.6$, 134.2, 134.5, 146.1. Anal. Calcd for $\mathrm{C}_{20} \mathrm{H}_{21} \mathrm{~N}_{3}: \mathrm{N}, 13.85$. Found: $\mathrm{N}, 13.83$.

\section{General procedure for the preparation of acetylenes $10 \mathbf{a}-\mathbf{k}$}

A stirred solution of $t$-BuOK $(0.90 \mathrm{~g}, 8.0 \mathrm{mmol})$ and 1,2-diaryl-1-(1H-1,2,3-benzotriazol1yl)ethane 9a-k $(4.0 \mathrm{mmol})$ in DMF $(8 \mathrm{~mL})$ was heated at $80{ }^{\circ} \mathrm{C}$ for $10 \mathrm{~min}$. The reaction mixture was cooled to $25^{\circ} \mathrm{C}$ and ice water was added. The obtained mixture was extracted with $\mathrm{Et}_{2} \mathrm{O}(3 \times 20 \mathrm{~mL})$, combined organic extracts were washed with water $(4 \times 20 \mathrm{~mL})$, dried over $\mathrm{MgSO}_{4}$, and evaporated. The crude product was purified on silica (EtOAc/Hexanes 1/3 as an eluent) to give the desired acetylenes $10 \mathbf{a}-\mathbf{k}$.

Phenyl-(p-tolyl)acetylene (10a). Yellow prisms (0.677 g, 88\%), mp 75-78 ${ }^{\circ} \mathrm{C}\left(\right.$ lit. $\left.\mathrm{mp}^{10 \mathrm{a}} 77-79{ }^{\circ} \mathrm{C}\right)$; ${ }^{1} \mathrm{H}$ NMR (300 MHz, $\left.\mathrm{CDCl}_{3}\right): \delta 2.36(\mathrm{~s}, 3 \mathrm{H}), 7.15$ (d, 2H, J = 7.8 Hz), 7.28-7.38 (m, 3H), 7.42 $(\mathrm{d}, 2 \mathrm{H}, J=7.8 \mathrm{~Hz}), 7.46-7.56(\mathrm{~m}, 2 \mathrm{H}) .{ }^{13} \mathrm{C} \mathrm{NMR}\left(75 \mathrm{MHz}, \mathrm{CDCl}_{3}\right): \delta 21.5,88.7,89.5,120.2$, 123.5, 128.1, 128.3, 129.1, 131.5, 131.6, 138.4. Anal. Calcd for $\mathrm{C}_{15} \mathrm{H}_{12}$ (192.26): C, 93.71; $\mathrm{H}$, 6.29; Found: C, 93.38; H, 6.70 .

Diphenylacetylene (10b). White prisms $(0.456 \mathrm{~g}, 64 \%), \mathrm{mp} 59-61$ (lit. mp $\left.{ }^{8 \mathrm{~b}} 58-61{ }^{\circ} \mathrm{C}\right) ;{ }^{1} \mathrm{H}$ NMR (300 MHz, $\left.\mathrm{CDCl}_{3}\right): \delta 7.31-7.34(\mathrm{~m}, 6 \mathrm{H}), 7.53(\mathrm{~d}, 4 \mathrm{H}, J=4.8 \mathrm{~Hz}) .{ }^{13} \mathrm{C} \mathrm{NMR}(75 \mathrm{MHz}$, $\left.\mathrm{CDCl}_{3}\right): \delta 89.3,123.2,128.2,128.3,131.6$. Anal. Calcd for $\mathrm{C}_{14} \mathrm{H}_{10}(178.24): \mathrm{C}, 94.34 ; \mathrm{H}, 5.66$; Found: C, 94.39; H, 6.03.

Phenyl(4-nitrophenyl)acetylene (10c). Yellow prisms $(0.750 \mathrm{~g}, 84 \%), \mathrm{mp} 113-114{ }^{\circ} \mathrm{C}$; ${ }^{1} \mathrm{H}$ NMR $\delta 7.38-7.42(\mathrm{~m}, 3 \mathrm{H}), 7.55-7.58(\mathrm{~m}, 2 \mathrm{H}), 7.67(\mathrm{~d}, 2 \mathrm{H}, J=8.7 \mathrm{~Hz}), 8.22(\mathrm{~d}, 2 \mathrm{H}, J=8.4 \mathrm{~Hz})$, ${ }^{13} \mathrm{C}$ NMR $\delta 87.5,94.7,122.1,123.6,128.5,129.3,130.2,131.8,132.2,146.9$. Calcd for $\mathrm{C}_{14} \mathrm{H}_{9} \mathrm{NO}_{2}$ (223.23): N, 6.27; Found: N, 6.10.

Di-p-tolylacetylene (10d). Yellow prisms (0.726 g, 88\%), mp 122-123 ${ }^{\circ} \mathrm{C}$ (lit. mp ${ }^{18 \mathrm{c}} 123-124{ }^{\circ} \mathrm{C}$ ). ${ }^{1} \mathrm{H}$ NMR (300 MHz, $\left.\mathrm{CDCl}_{3}\right): \delta 2.36(\mathrm{~s}, 6 \mathrm{H}), 7.14(\mathrm{~d}, 4 \mathrm{H}, J=7.8 \mathrm{~Hz}), 7.41(\mathrm{~d}, 4 \mathrm{H}, J=8.1 \mathrm{~Hz})$. ${ }^{13} \mathrm{C}$ NMR $\left(75 \mathrm{MHz}, \mathrm{CDCl}_{3}\right): \delta 21.5,88.8,120.4,129.1,131.4,138.2$.

1-(p-Tolylethynyl)naphthalene (10e). Yellow prisms $(0.843 \mathrm{~g}, 87 \%), \mathrm{mp} 55-56{ }^{\circ} \mathrm{C}$ (lit. $\mathrm{mp}^{8 \mathrm{~d}}$ 55-56 $\left.{ }^{\circ} \mathrm{C}\right) ;{ }^{1} \mathrm{H}$ NMR $\left(300 \mathrm{MHz}, \mathrm{CDCl}_{3}\right): \delta 2.50(\mathrm{~s}, 3 \mathrm{H}), 7.32(\mathrm{~d}, 2 \mathrm{H}, J=8.1 \mathrm{~Hz}), 7.46-7.60(\mathrm{~m}$, $1 \mathrm{H}), 7.61-7.68(\mathrm{~m}, 4 \mathrm{H}), 7.90(\mathrm{~d}, 1 \mathrm{H}, J=7.2 \mathrm{~Hz}), 7.90-8.05(\mathrm{~m}, 2 \mathrm{H}), 8.62(\mathrm{~d}, 1 \mathrm{H}, J=8.4 \mathrm{~Hz})$. ${ }^{13} \mathrm{C}$ NMR $\left(75 \mathrm{MHz}, \mathrm{CDCl}_{3}\right): \delta 21.5,86.9,94.5,120.3,121.1,125.2,126.2,126.3,126.7,128.2$, 128.5, 129.1, 130.1, 131.5, 133.1, 133.2, 138.5. Anal. Calcd for $\mathrm{C}_{19} \mathrm{H}_{14}$ (242.32): C, 94.18; $\mathrm{H}$, 5.82. Found: C, 93.90; H, 6.03.

2-(p-Tolylethynyl)pyridine (10f). Yellow prisms $(0.495 \mathrm{~g}, 64 \%), \mathrm{mp} 60-61{ }^{\circ} \mathrm{C} ;{ }^{1} \mathrm{H}$ NMR (300 $\left.\mathrm{MHz}, \mathrm{CDCl}_{3}\right): \delta 2.38(\mathrm{~s}, 3 \mathrm{H}), 7.17(\mathrm{~d}, 1 \mathrm{H}, J=7.8 \mathrm{~Hz}), 7.20-7.27(\mathrm{~m}, 2 \mathrm{H}), 7.43-7.54(\mathrm{~m}, 3 \mathrm{H})$, $7.67\left(\mathrm{td}, 1 \mathrm{H}, J_{1}=7.8 \mathrm{~Hz}, J_{2}=1.8 \mathrm{~Hz}\right), 7.62(\mathrm{~d}, 1 \mathrm{H}, J=4.5 \mathrm{~Hz}) \cdot{ }^{13} \mathrm{C} \mathrm{NMR}\left(75 \mathrm{MHz}, \mathrm{CDCl}_{3}\right): \delta$ $21.4,89.0,89.4,119.0,122.5,126.9,129.0,131.8,136.0,139.1,143.5,149.9$. Anal. Calcd for $\mathrm{C}_{14} \mathrm{H}_{11} \mathrm{~N}$ (193.25): N, 7.25. Found: N, 7.45.

2-Furyl(4-methylphenyl)acetylene (10g). Yellow oil $(0.452 \mathrm{~g}, 62 \%) ;{ }^{1} \mathrm{H}$ NMR (300 MHz, $\left.\mathrm{CDCl}_{3}\right): \delta 2.37(\mathrm{~s}, 3 \mathrm{H}), 6.42\left(\mathrm{dd}, 1 \mathrm{H}, J_{1}=3.0 \mathrm{~Hz} ; J_{2}=1.8 \mathrm{~Hz}\right), 6.63(\mathrm{~d}, 1 \mathrm{H}, J=3.6 \mathrm{~Hz}), 7.15(\mathrm{~d}$, $2 \mathrm{H}, J=8.1 \mathrm{~Hz}), 7.41(\mathrm{~d}, 1 \mathrm{H}, J=2.4 \mathrm{~Hz}), 7.42(\mathrm{~d}, 2 \mathrm{H}, J=3.6 \mathrm{~Hz}) .{ }^{13} \mathrm{C} \mathrm{NMR}\left(75 \mathrm{MHz}, \mathrm{CDCl}_{3}\right)$ : 
$\delta 21.5,78.7,93.4,111.0,114.9,119.1,129.1,131.3,137.3,138.9,143.4$. HRMS EI for $\mathrm{C}_{13} \mathrm{H}_{10} \mathrm{O}$ $\left[\mathrm{M}^{+}\right]$182.0732 Found: 182.0726 .

2-(p-Tolylethynyl)thiophene (10h). Yellow prisms (0.603 g, 76\%), mp 58-59 ${ }^{\circ} \mathrm{C} ;{ }^{1} \mathrm{H}$ NMR $\left(300 \mathrm{MHz}, \mathrm{CDCl}_{3}\right): \delta 2.36(\mathrm{~s}, 1 \mathrm{H}), 7.00(\mathrm{t}, 1 \mathrm{H}, J=4.5 \mathrm{~Hz}), 7.15(\mathrm{~d}, 2 \mathrm{H}, J=7.8 \mathrm{~Hz}), 7.22-7.28$ $(\mathrm{m}, 2 \mathrm{H}), 7.40(\mathrm{~d}, 2 \mathrm{H}, J=7.8 \mathrm{~Hz}) .{ }^{13} \mathrm{C} \mathrm{NMR}\left(75 \mathrm{MHz}, \mathrm{CDCl}_{3}\right): \delta 21.5,81.9,93.2,119.8,123.5$, 127.0, 127.0, 129.1, 131.3, 131.6, 138.6. Anal. Calcd for $\mathrm{C}_{13} \mathrm{H}_{10} \mathrm{~S}$ (198.29): C, 78.75; H, 5.08; Found: C, 78.63; H, 5.46 .

(4-Chlorophenyl)phenylacetylene (10i). White prisms $(0.408 \mathrm{~g}, 48 \%), \mathrm{mp} 80-81{ }^{\circ} \mathrm{C}$ (lit. $\mathrm{mp}^{18 \mathrm{e}}$ 81-82 $\left.{ }^{\circ} \mathrm{C}\right) ;{ }^{1} \mathrm{H}$ NMR $\left(300 \mathrm{MHz}, \mathrm{CDCl}_{3}\right): \delta 7.27-7.39(\mathrm{~m}, 5 \mathrm{H}), 7.42-7.49(\mathrm{~m}, 2 \mathrm{H}), 7.49-7.56$ (m, 2H). ${ }^{13} \mathrm{C}$ NMR $\left(75 \mathrm{MHz}, \mathrm{CDCl}_{3}\right): \delta 88.2,90.3,121.7,122.9,128.4,128.4,128.6,131.5$, 132.8, 134.2. Anal. Calcd for $\mathrm{C}_{14} \mathrm{H}_{9} \mathrm{Cl}$ (212.68): C, 79.06; H, 4.27; Found: C, 78.93; H, 4.23.

1-[2-(4-Chlorophenyl)ethynyl]naphthalene (10j). White prisms $(0.788 \mathrm{~g}, 75 \%), \mathrm{mp} 84-85{ }^{\circ} \mathrm{C}$; ${ }^{1} \mathrm{H}$ NMR $\delta\left(300 \mathrm{MHz}, \mathrm{CDCl}_{3}\right): 7.35(\mathrm{~d}, 2 \mathrm{H}, J=8.4 \mathrm{~Hz}), 7.40-7.66(\mathrm{~m}, 5 \mathrm{H}), 7.74(\mathrm{~d}, 1 \mathrm{H}, J=6.9$ $\mathrm{Hz}), 7.78-7.90(\mathrm{~m}, 2 \mathrm{H}), 8.39(\mathrm{~d}, 1 \mathrm{H}, J=8.4 \mathrm{~Hz}) .{ }^{13} \mathrm{C} \mathrm{NMR}\left(75 \mathrm{MHz}, \mathrm{CDCl}_{3}\right): \delta 88.5,93.1$, 120.5, 121.8, 125.2, 126.1, 126.5, 126.8, 128.3, 128.7, 129.0, 130.5, 132.8, 133.2, 134.3. Anal. Calcd for $\mathrm{C}_{18} \mathrm{H}_{11} \mathrm{Cl}$ (262.74): C, 82.29; H, 4.22; Found: C, 82.13; H, 4.29 .

3-[2-(4-Chlorophenyl)ethynyl]pyridine (10k). White prisms $(0.752 \mathrm{~g}, 88 \%), \mathrm{mp} 82-83{ }^{\circ} \mathrm{C} ;{ }^{1} \mathrm{H}$ NMR (300 MHz, CDCl $): \delta 7.22-7.42(\mathrm{~m}, 3 \mathrm{H}), 7.48(\mathrm{~d}, 2 \mathrm{H}, J=8.4 \mathrm{~Hz}), 7.81(\mathrm{~d}, 1 \mathrm{H}, J=8.1$ $\mathrm{Hz}), 7.57(\mathrm{~d}, 1 \mathrm{H}, J=4.8 \mathrm{~Hz}), 8.76(\mathrm{~s}, 1 \mathrm{H}) .{ }^{13} \mathrm{C} \mathrm{NMR}\left(75 \mathrm{MHz}, \mathrm{CDCl}_{3}\right): \delta 86.9,91.4,120.1$, 121.0, 123.0, 128.8, 132.9, 134.9, 138.4, 148.8, 152.2. Anal. Calcd for $\mathrm{C}_{13} \mathrm{H}_{8} \mathrm{ClN}$ (213.67): C, 73.08; H, 3.77; N, 6.56. Found: C, 72.95; H, 3.65; N, 6.48 .

\section{Acknowledgements}

We thank Dr. Christophe Chassaing, Dr. Stewart Barrow and Dr. Zhenghong Zhang for their help in the early stage of this research project.

\section{References}

1. (a) The Chemistry of the Carbon-Carbon Triple Bond; Patai, S., Ed.; J. Wiley: New York, 1978. (b) Brandsma, L. In Preparative Acetylenic Chemistry; Elsevier: New York, 1988.

2. (a) Nelson, J. C.; Young, J. K.; Moore, J. S. J. Org. Chem. 1996, 61, 8160. (b) Jones, II, L.; Schumm, J. S.; Tour, J. M. J. Org. Chem. 1997, 62, 1388. (c) Siemsen, P.; Livingston, R. C.; Dieberich, F. Angew. Chem., Int. Ed. 2000, 39, 2632. (d) Huang, S.; Tour, J. M. J. Org. Chem. 1999, 64, 8898.

3. Danel, K.; Lin, J. T. ARKIVOC 2002, (i), 12. 
4. (a) Takahashi, T.; Shen, B.; Nakajima, K.; Xi, Z. J. Org. Chem. 1999, 64, 8706. (b) Larock, R. C.; Tian, Q. J. Org. Chem. 1998, 63, 2002. (c) Harrity, J. P. A.; Kerr, W. J. Tetrahedron Lett. 1993, 34, 2995. (d) Larock, R. C.; Doty, M. J. J. Org. Chem. 1993, 58, 4579.

5. (a) Gotthardt, H.; Huss, O. M. Tetrahedron Lett. 1978, 38, 3617. (b) Nan, Y.; Miao, H.; Yang, Z. Org. Lett. 2000, 2, 279. (c) Yoneda, E.; Sugioka, T.; Hirao, K.; Zhang, S.-W.; Takahashi, S. J. Chem. Soc., Perkin Trans. 1 1998, 477. (d) Kondo, Y.; Shiga, F.; Murata, N.; Sakamoto, T.; Yamanaka, H. Tetrahedron 1994, 50, 11803. (e) Rodriguez, A. L.; Koradin, C.; Dohle, W.; Knochel, P. Angew. Chem., Int. Ed. 2000, 39, 2488. (f) Yasuhara, A.; Kanamori, Y.; Kaneko, M.; Numata, A.; Kondo, Y.; Sakamoto, T. J. Chem. Soc., Perkin Trans. 1 1999, 529. (g) Jiang, B.; Tilley, T. D. J. Am. Chem. Soc. 1999, 121, 9744. (h) Dzhemilev, U. M.; Selimov, F. A.; Tolstikov, G. A. ARKIVOC 2001, (ix), 85.

6. (a) Stieber, F.; Grether, U.; Waldmann, H. Angew. Chem., Int. Ed. 1999, 38, 1073. (b) Yu, K.-L.; Deshpande, M. S.; Vyas, D. M. Tetrahedron Lett. 1994, 35, 8919. (c) Kang, S.-K.; Kim, J.-S.; Yoon, S.-K.; Lim, K.-H.; Yoon, S. S. Tetrahedron Lett. 1998, 39, 3011. (d) Zhang, H.-C.; Brumfield, K. K.; Jaroskova, L.; Maryanoff, B. E. Tetrahedron Lett. 1998, 39, 4449. (e) Young, J. K.; Nelson, J. C.; Moore, J. S. J. Am. Chem. Soc. 1994, 116, 10841.

7. Kalinin, V. N. Synthesis 1992, 413.

8. (a) Coleman, G. H.; Maxwell, R. D. J. Am. Chem. Soc. 1934, 56, 132. (b) Coleman, G. H.; Holst, W. H.; Maxwell, R. D. J. Am. Chem. Soc. 1936, 58, 2310.

9. Kodomari, M.; Nagaoka, T.; Furusawa, Y. Tetrahedron Lett. 2001, 42, 3105.

10. (a) Katritzky, A. R.; Gordeev, M. F. J. Chem. Soc., Perkin Trans. 1 1992, 1295. (b) Paventi, M.; Elce, E.; Jackman, R. J.; Hay, A. S. Tetrahedron Lett. 1992, 33, 6405.

11. (a) Kang, S.-K.; Yoon, S.-K.; Kim, Y.-M. Org. Lett. 2001, 3, 2697. (b) Kitamura, T.; Tanaka, T.; Taniguchi, H.; Stang, P. J. J. Chem. Soc., Perkin Trans. 1 1991, 2892.

12. (a) Arcadi, A.; Cacchi, S.; Marinelli, F. Tetrahedron Lett. 1989, 30, 2581. (b) Alvarez, A.; Guzman, A.; Ruiz, A.; Velarde, E. J. Org. Chem. 1992, 57, 1653. (c) De la Rosa, M. A.; Velarde, E.; Guzman, A. Synth. Commun. 1990, 20, 2059.

13. Chang, S.; Yang, S. H.; Lee, P. H. Tetrahedron Lett. 2001, 42, 4833.

14. Kang, S.-K.; Ryu, H.-C.; Hong, Y.-T. J. Chem. Soc., Perkin Trans. 1 2001, 736.

15. Han, X.; Hartmann, G. A.; Brazzale, A.; Gaston, R. D. Tetrahedron Lett. 2001, 42, 5837.

16. Katritzky, A. R.; Lan, X.; Yang, J. Z.; Denisko, O. V. Chem. Rev. 1998, 98, 409

17. Sardarian, A. R.; Kaboudin, B. Synth. Commun. 1997, 27, 543.

18. (a) Abramov, V. S.; Semenova, N. A. J. Gen. Chem. USSR, Eng. Transl. 1958, 28, 3087. (b) Coleman, G. H.; Holst, W. H.; Maxwell, R. D. J. Am. Chem. Soc. 1936, 58, 2310. (c) Coleman, G. H.; Maxwell, R. D. J. Am. Chem. Soc. 1934, 56, 132. (d) Kasahara, A.; Izumi, T.; Shimizu, I.; Satou, M.; Katou, T. Bull. Chem. Soc. Jap. 1982, 55, 2434. (e) Colvin, E. W.; Hamill, B. J. J. Chem. Soc., Perkin Trans. 1 1977, 869. 